\title{
Sensitivity, specificity, inclusivity and exclusivity of the updated Aptima Combo 2 assay, which provides detection coverage of the new diagnostic-escape Chlamydia trachomatis variants
}

Magnus Unemo ${ }^{1 *}$ D, Marit Hansen ${ }^{1}$, Ronza Hadad ${ }^{1}$, Mirja Puolakkainen², Henrik Westh ${ }^{3,4}$, Kaisu Rantakokko-Jalava ${ }^{5}$, Carina Thilesen ${ }^{6}$, Michelle J. Cole ${ }^{7}$, Iryna Boiko ${ }^{1,8}$, Pham T. Lan ${ }^{9}$, Daniel Golparian ${ }^{1}$, Shin Ito $^{10}$ and Martin Sundqvist ${ }^{1}$

\begin{abstract}
Background: Four new variants of Chlamydia trachomatis (nvCTs), detected in several countries, cause falsenegative or equivocal results using the Aptima Combo 2 assay (AC2; Hologic). We evaluated the clinical sensitivity and specificity, as well as the analytical inclusivity and exclusivity of the updated AC2 for the detection of CT and Neisseria gonorrhoeae (NG) on the automated Panther system (Hologic).

Methods: We examined 1004 clinical AC2 samples and 225 analytical samples spiked with phenotypically and/or genetically diverse NG and CT strains, and other potentially cross-reacting microbial species. The clinical AC2 samples included CT wild type (WT)-positive $(n=488)$, all four described AC2 diagnostic-escape nvCTs $(n=170)$, NGpositive $(n=214)$, and CT/NG-negative $(n=202)$ specimens.

Results: All nvCT-positive samples (100\%) and 486 (99.6\%) of the CT WT-positive samples were positive in the updated AC2. All NG-positive, CT/NG-negative, Trichomonas vaginalis (TV)-positive, bacterial vaginosis-positive, and Candida-positive AC2 specimens gave correct results. The clinical sensitivity and specificity of the updated AC2 for CT detection was 99.7 and 100\%, respectively, and for NG detection was 100\% for both. Examining spiked samples, the analytical inclusivity and exclusivity were $100 \%$, i.e., in clinically relevant concentrations of spiked microbe.

Conclusions: The updated AC2, including two CT targets and one NG target, showed a high sensitivity, specificity, inclusivity and exclusivity for the detection of $C T W T, n v C T s$, and NG. The updated AC2 on the fully automated Panther system offers a simple, rapid, high-throughput, sensitive, and specific diagnosis of $C T$ and NG, which can easily be combined with detection of Mycoplasma genitalium and TV.
\end{abstract}

Keywords: Finnish new variant, Chlamydia trachomatis, Aptima combo 2, 235 rRNA, Validation, Surveillance

\footnotetext{
* Correspondence: magnus_unemo@yahoo.com

${ }^{1}$ World Health Organization Collaborating Centre for Gonorrhoea and Other Sexually Transmitted Infections (STIs), National Reference Laboratory for STIs, Department of Laboratory Medicine, Clinical Microbiology, Faculty of Medicine and Health, Örebro University, SE-701 85 Örebro, Sweden

Full list of author information is available at the end of the article
}

C C The Author(s). 2020 Open Access This article is licensed under a Creative Commons Attribution 4.0 International License, which permits use, sharing, adaptation, distribution and reproduction in any medium or format, as long as you give appropriate credit to the original author(s) and the source, provide a link to the Creative Commons licence, and indicate if changes were made. The images or other third party material in this article are included in the article's Creative Commons licence, unless indicated otherwise in a credit line to the material. If material is not included in the article's Creative Commons licence and your intended use is not permitted by statutory regulation or exceeds the permitted use, you will need to obtain permission directly from the copyright holder. To view a copy of this licence, visit http://creativecommons.org/licenses/by/4.0/ The Creative Commons Public Domain Dedication waiver (http://creativecommons.org/publicdomain/zero/1.0/) applies to the data made available in this article, unless otherwise stated in a credit line to the data. 


\section{Background}

Curable sexually transmitted infections (STIs) remain major global public health concerns [1]. Chlamydia trachomatis (CT) infection is the most common bacterial STI with 127 million estimated cases among adults each year globally [1]. In the European Union/European Economic Area (EU/EEA) and in many other international settings, the incidence of CT infections has increased during the past decade $[1,2]$. The widespread use of highly sensitive and specific nucleic acid amplification tests (NAATs), particularly in high-income countries, has substantially contributed to the increased detection and incidence. Undetected and untreated CT infections can result in serious complications and sequelae, including infertility. CT infections are frequently asymptomatic and accordingly not detected if appropriate laboratory diagnostics is not performed [3].

Validated and quality-assured CT NAATs are recommended for highly sensitive and specific diagnosis of CT infections. Compared to other CT diagnostic methods, NAATs have highly superior sensitivities with maintained high specificities, use non-invasive specimens, are rapid, and provide opportunities for automation [3]. Initially, CT was considered to be genomically highly conserved. However, in 2006 the Swedish new variant of CT (nvCT) was described [4]. This Swedish nvCT has a deletion in the cryptic plasmid resulting in false negative results using the Roche and Abbott NAATs available at that time $[5,6]$. Subsequently, whole genome sequencing of CT provided evidence of relatively substantial recombination and a mutational frequency level similar to the one in many other bacterial species [7, 8]. This showed that CT evolves more than previously predicted and diagnostic-escape CT mutants can emerge.

The sensitivity and specificity of the US FDAapproved Aptima Combo 2 assay (AC2; Hologic Inc., San Diego, CA, USA), based on target capture (TC) and transcription-mediated amplification (TMA) chemistries, for detection of CT (target: 23S rRNA) and Neisseria gonorrhoeae (NG; target: 16S rRNA) have proven excellent in many studies [9-17]. However, in early 2019, the Finnish nvCT (FI-nvCT) was identified [18, 19]. This variant has a single nucleotide polymorphism (SNP) in the CT 23S rRNA gene, i.e. C1515T (Escherichia coli numbering), resulting in escaped detection by the acridinium ester CT detection probe used in AC2 [18-24]. The FI-nvCT was initially estimated to represent $6-10 \%$ of the CT positive samples in Finland [18, 19]. The FInvCT has subsequently been reported in Sweden [20], Norway [21], and Denmark [24]. Three additional AC2 diagnostic-escape nvCTs have been reported in single specimens in Norway [21], the United Kingdom [25], and Japan (the present study), and one of these three nvCTs was found widely spread in Denmark [24]. These three AC2 diagnostic-escape nvCTs, as the FI-nvCT, have a SNP in the AC2 CT probe detection sequence of $23 \mathrm{~S}$ rRNA, i.e. 23S rRNA C1514T, G1523A [21, 24, 25], or C1522T (the present study). The Aptima C. trachomatis NAAT (ACT; Hologic Inc.), which targets CT 16S rRNA, detects all of these diagnostic-escape nvCTs. Reflex testing of AC2 samples with relative light unit (RLU) values of 15-99 (mostly "high negative" or equivocal results) using ACT was implemented in MayJune 2019 in many European countries [22, 26]. However, this ACT reflex testing substantially increases the work load and associated costs in these laboratories, and a more sustainable solution is imperative. Accordingly, an updated version of $\mathrm{AC} 2$, which was designed to detect also all these and other diagnostic-escape nvCTs, has now been developed by Hologic Inc. (San Diego, USA). This updated AC2 assay includes one additional $\mathrm{CT}$ detection probe targeting a second region of the CT $23 \mathrm{~S}$ rRNA [27].

The aim of the present study was to evaluate the clinical sensitivity and specificity, as well as the analytical inclusivity and exclusivity of the updated AC2 (provided as research-use-only [RUO] material) for detection of wildtype (WT) CT, nvCTs, and NG on the automated Panther system (Hologic).

\section{Methods}

The evaluation panel consisted of 1004 clinical, routinely collected samples (from 2015 to 2020) previously diagnosed using AC2 and/or ACT, in accordance with the instructions from the manufacturer (Hologic), and 225 samples spiked with geographically and temporally (from 1971 to 2016) diverse isolates of NG, CT, FI-nvCT, and T. vaginalis (TV), as well as non-NG Neisseria, Moraxella, non-TV Trichomonas, or non-CT Chlamydia.

\section{Clinical NAAT samples}

The AC2 clinical samples consisted of CT WT-positive specimens $(\mathrm{n}=488 ; 84$ of these were also positive for NG); diagnostic-escape nvCTs (FI-nvCT $[\mathrm{n}=114]$, nvCT-G1523A [ $\mathrm{n}=46$; one positive also for NG], nvCTC1522T [n=6], and nvCT-C1514T [n=4]; all verified by $23 \mathrm{~S}$ rRNA gene sequencing or the FI-nvCT TMA assay [23]); NG-positive specimens ( $\mathrm{n}=214 ; 84$ of these were also positive for CT); CT- and NG-negative specimens $(n=202)$; and specimens positive for TV $(n=5)$, bacterial vaginosis $(n=5)$, and Candida species $(n=5)$. The nvCT-samples comprised mainly first-void urine or vaginal/cervical samples from Finland, Sweden, Norway, Denmark, the United Kingdom, and Japan. Prior to testing with the updated AC2 assay, all clinical AC2 samples were collected and stored $\left(-20-70{ }^{\circ} \mathrm{C}\right)$ in routine diagnostics (standard care), and no patient identification information was available in the present study. All clinical 
AC2 samples were collected in Aptima Urine Specimen Transport Tubes or Aptima Vaginal Swab Specimen Collection Kit (Hologic). Results from the initial routine testing using the original version of $\mathrm{AC} 2$ were used for comparison against the updated AC2 results.

\section{Analytical inclusivity and exclusivity panel}

The panel was assembled to include phenotypically and/ or genetically diverse strains. For CT inclusivity, all nine main genotypes, including L1-L3 causing Lymphogranuloma venereum (LGV), and variants such as the Swedish nvCT [4-6], were represented. Furthermore, 20 samples including in vitro transcript $(5 \mathrm{fg}$ ) of the FI-nvCT $23 \mathrm{~S}$ rRNA (Panel G; Hologic) were examined. The four nonCT Chlamydia species C. muridarium, C. suis, C. psittaci, and $C$. pneumoniae were initially tested in high concentration $\left(\sim 1.1 \times 10^{7}\right.$ genome equivalents (GEQs)/test) to challenge the exclusivity. For NG inclusivity, the geographically, temporally and genomically diverse 2016 WHO reference strains $(\mathrm{n}=14)$ [28], which include prolyliminopeptidase (PIP), porA pseudogene, and $с p p B \mathrm{mu}-$ tants that have previously escaped diagnostics using other NAATs, were examined. Due to the high level of genetic homogeneity between NG and non-NG Neisseria species and problems with cross-reactivity in several NG NAATs [13], a high number of different strains $(\mathrm{n}=151)$ representing 14 diverse non-NG Neisseria and three Moraxella species were tested in high concentration $\left(\sim 2 \times 10^{7}\right.$ genome equivalents (GEQs)/test) to substantially challenge the exclusivity. Finally, T. vaginalis samples $(\mathrm{n}=10)$, including the two ATCC strains 30,001 and 50,140, and 10 samples including four different non- $T$. vaginalis Trichomonas species were tested in a high concentration ( $1.25 \times 10^{7} \mathrm{GEQ} /$ test). All the preparation and testing of spiked samples were performed as previously described [29]. If any cross-reactivity was identified, the crossreacting sample was diluted to more clinically relevant concentrations, i.e. the sample was diluted to contain similar number of GEQs as CT and NG are present in within strongly CT and NG positive samples [30-32].

\section{NAAT testing}

Like the original AC2 formulation, the updated AC2 assay is based on TC and TMA chemistries. Testing with the updated AC2 (provided as RUO material) was performed on the Panther system (Hologic), in strict accordance with the instructions from the manufacturer. All testing was performed blinded in regards to the results from other testing.

\section{Results}

The results of all testing of AC2 clinical samples and spiked samples with the updated AC2 are summarised in Table 1.

\section{Clinical NAAT samples}

All 170 nvCT-positive samples (FI-nvCT [ $\mathrm{n}=114]$, nvCT-G1523A [n=46], nvCT-C1522T [n=6], and nvCT-C1514T $[\mathrm{n}=4]$ ) and $486(99.6 \%)$ of the $488 \mathrm{CT}$ WT-positive samples were positive in the updated AC2. The two CT WT-positive samples that were negative in the updated AC2 showed RLU values of 14 and 8 in the updated AC2, while they were positive in the original AC2 (RLU values: 837 and 1215). All NG-positive ( $\mathrm{n}=$ 214), CT/NG-negative $(\mathrm{n}=202), \quad T$. vaginalis-positive $(\mathrm{n}=5)$, bacterial vaginosis-positive $(\mathrm{n}=5)$, and Candidapositive $(n=5)$ AC2 specimens gave correct results with the updated AC2 (Table 1). The overall clinical sensitivity and specificity of the updated AC2 for CT detection was 99.7\% (CI 95\%: 98.9-100\%) and 100\% (CI 95\%: 98.9-100\%), respectively. The overall clinical sensitivity and specificity for NG detection was 100\% (CI 95\%: 98.3-100\%) and 100\% (CI 95\%: 99.5-100\%), respectively.

\section{Analytical inclusivity and exclusivity panel}

The inclusivity and exclusivity panel included samples spiked with the following: nine CT strains of different genotypes $(n=9)$ and the Swedish nvCT $(n=1)$; in vitro transcript of the FI-nvCT 23S rRNA $(\mathrm{n}=20)$; four nonCT Chlamydia species $(\mathrm{n}=10)$; all 2016 WHO NG reference strains $(\mathrm{n}=14) ; 151$ strains of non-NG Neisseria species $(\mathrm{n}=14)$ and Moraxella species $(\mathrm{n}=3) ;$ T. vaginalis samples, including the two ATCC strains 30,001 and $50,140(\mathrm{n}=10)$; and five reference strains of four non- $T$. vaginalis Trichomonas species $(\mathrm{n}=10)$. In clinically relevant concentrations, all samples gave correct results using the updated AC2 (Table 1).

Notably, in high concentrations $\left(\sim 2 \times 10^{7}\right.$ GEQs/test), two $N$. elongata strains were repeatedly yielding equivocal NG results with the updated AC2 (RLU values: 86114 and 71-118, respectively). In clinically more relevant concentrations $\left(\sim 2 \times 10^{5} \mathrm{GEQs} /\right.$ test $)$, both strains were negative. Furthermore, in high concentrations $(\sim 1.1 \times$ $10^{7}$ GEQs/test) C. suis (ATCC VR-1474) and C. muridarum (ATCC VR-123) tested CT positive with the updated AC2 (RLU values: 951 and 1159, respectively). However, in clinically more relevant concentrations $(\sim$ $1.1 \times 10^{5}$ GEQs/test) both were negative (Table 1) and none of these animal pathogens have to our knowledge been found in human.

\section{Discussion}

The present study is the first clinical and analytical validation of the updated AC2 NAAT (Hologic), which includes one NG target and two CT targets [27] and most recently was granted US FDA approval (https://www.accessdata.fda.gov/ scripts/cdrh/cfdocs/cfPMN/pmn.cfm?ID=K200866). Since the initial report of the FI-nvCT in late-May 2019 in Finland $[18,19]$ and subsequent reports of FI-nvCT and additional 
Table 1 Clinical and analytical detection of the updated Aptima Combo 2 assay (AC2; detecting CT and NG), which provides detection coverage of all diagnostic-escape new variants of $\mathrm{CT}$ [18-25, the present study]

\begin{tabular}{|c|c|c|c|c|c|}
\hline \multirow[t]{2}{*}{ Clinical specimen } & \multirow{2}{*}{$\begin{array}{l}\text { Number } \\
\text { tested }\end{array}$} & \multicolumn{2}{|c|}{ Positive AC2 } & \multicolumn{2}{|c|}{ Positive updated AC2 } \\
\hline & & $\overline{\mathrm{CT}}$ & NG & $\overline{\mathrm{CT}}$ & NG \\
\hline CT wild type (C1515)-positive clinical AC2 specimens ${ }^{a, b}$ & 488 & 488 & 84 & 486 & 84 \\
\hline FI-nvCT (C1515T)-positive clinical AC2 specimens ${ }^{\text {b }}$ & 114 & 0 & 0 & 114 & 0 \\
\hline nvCT-G1523A-positive clinical AC2 specimens ${ }^{a, b}$ & 46 & 0 & 1 & 46 & 1 \\
\hline nvCT-C1522T-positive clinical AC2 specimens ${ }^{b}$ & 6 & 0 & 0 & 6 & 0 \\
\hline nvCT-C1514T-positive clinical AC2 specimens ${ }^{b}$ & 4 & 0 & 0 & 4 & 0 \\
\hline N. gonorrhoeae-positive, CT-negative AC2 specimens & 129 & 0 & 129 & 0 & 129 \\
\hline T. vaginalis-positive AC2 specimens & 5 & 0 & 0 & 0 & 0 \\
\hline Bacterial vaginosis-positive AC2 specimens & 5 & 0 & 0 & 0 & 0 \\
\hline Candida spp.-positive AC2 specimens & 5 & 0 & 0 & 0 & 0 \\
\hline C. trachomatis and N. gonorrhoeae-negative clinical AC2 specimens & 202 & 0 & 0 & 0 & 0 \\
\hline \multicolumn{6}{|l|}{ Microbial species in spiked specimens } \\
\hline C. trachomatis $\mathrm{Ba}, \mathrm{D}, \mathrm{E}, \mathrm{F}, \mathrm{G}, \mathrm{H}, \mathrm{J}, \mathrm{K}, \mathrm{L} 2 \mathrm{~b}$ & 9 & 9 & 0 & 9 & 0 \\
\hline C. trachomatis SE-nvCT [4-6] & 1 & 1 & 0 & 1 & 0 \\
\hline FI-nvCT $23 \mathrm{~S}$ rRNA in vitro transcript & 20 & 0 & 0 & 20 & 0 \\
\hline C. suis (ATCC VR-1474) ${ }^{C}$ & 2 & 0 & 0 & 0 & 0 \\
\hline C. muridarum (ATCC VR-123) ${ }^{c}$ & 2 & 0 & 0 & 0 & 0 \\
\hline C. pneumoniae (ATCC VR-2282, MBC011) & 4 & 0 & 0 & 0 & 0 \\
\hline C. psittaci (MBC013) & 2 & 0 & 0 & 0 & 0 \\
\hline Neisseria gonorrhoeae 2016 WHO reference strains [28] & 14 & 0 & 14 & 0 & 14 \\
\hline N. animalis & 1 & 0 & 0 & 0 & 0 \\
\hline N. bergeri & 1 & 0 & 0 & 0 & 0 \\
\hline N. cinerea & 9 & 0 & 0 & 0 & 0 \\
\hline N. elongata & 3 & 0 & 0 & 0 & 0 \\
\hline N. flava & 1 & 0 & 0 & 0 & 0 \\
\hline N. flavescens & 32 & 0 & 0 & 0 & 0 \\
\hline N. lactamica & 12 & 0 & 0 & 0 & 0 \\
\hline N. macacae & 17 & 0 & 0 & 0 & 0 \\
\hline N. mucosa & 17 & 0 & 0 & 0 & 0 \\
\hline N. oralis & 1 & 0 & 0 & 0 & 0 \\
\hline N. perflava & 22 & 0 & 0 & 0 & 0 \\
\hline N. sicca & 9 & 0 & 0 & 0 & 0 \\
\hline N. subflava & 6 & 0 & 0 & 0 & 0 \\
\hline N. meningitidis ${ }^{\mathrm{d}}$ & 17 & 0 & 0 & 0 & 0 \\
\hline Moraxella catarrhalis & 1 & 0 & 0 & 0 & 0 \\
\hline M. nonliquefaciens & 1 & 0 & 0 & 0 & 0 \\
\hline M. osloensis & 1 & 0 & 0 & 0 & 0 \\
\hline Trichomonas vaginalis (ATCC 30001, ATCC 50140) & 10 & 0 & 0 & 0 & 0 \\
\hline T. aotus (ATCC 50649) & 2 & 0 & 0 & 0 & 0 \\
\hline T. gallinae (ATCC $30002,30,230$ ) & 4 & 0 & 0 & 0 & 0 \\
\hline T. stableri (ATCC PRA-412) & 2 & 0 & 0 & 0 & 0 \\
\hline T. tenax (ATCC 30207) & 2 & 0 & 0 & 0 & 0 \\
\hline
\end{tabular}

AC2, Aptima Combo 2; CT, Chlamydia trachomatis; NG, Neisseria gonorrhoeae; FI-nvCT, Finnish new variant of CT; SE-nvCT, Swedish new variant of CT; ATCC, American Type Culture Collection; WHO, World Health Organization; RLU, relative light unit

a 84 samples and one sample, respectively, were also confirmed positive for NG

${ }^{\mathrm{b}}$ Confirmed by sequencing of the 23S rRNA gene or the FI-nvCT TMA assay [23]

${ }^{C}$ Positive in very high concentration, but negative in clinically more relevant concentrations

${ }^{d}$ Representing major meningococcal clones spreading worldwide, including serogroups $A, B, C, E, W, X, Y$ and $Z$ 
diagnostic-escape nvCTs in Sweden [20], Norway [21], Denmark [24], and the United Kingdom [25], reflex testing with $\mathrm{ACT}$ of possible $\mathrm{AC} 2$ diagnostic-escape nvCT has been performed in all European diagnostic laboratories [22, 26]. However, Hologic has now developed an updated version of the AC2 assay [27], which was designed to detect also all of the new diagnostic-escape nvCTs [18-25, the present study] and includes one additional CT detection probe targeting a second region of the CT $23 \mathrm{~S}$ rRNA. In the present study, we show that the updated AC2 assay has a maintained high clinical sensitivity and specificity, as well as an ideal analytical inclusivity and exclusivity, when examining samples spiked in clinically relevant concentrations of microbe, in the detection of WT CT and NG. Furthermore, the updated AC2 assay also effectively detected all of the described $\mathrm{AC} 2$ diagnosticescape nvCTs [18-25, the present study], i.e. clinical AC2 specimens containing the FI-nvCT $(\mathrm{n}=114)$, nvCT-G1523A $(\mathrm{n}=46)$, nvCT-C1522T $(\mathrm{n}=6)$, and nvCT-C1514T $(\mathrm{n}=4)$ were all positive. Thus, the clinical sensitivity and specificity of the updated AC2 for CT detection was 99.7 and 100\%, respectively. Despite that samples were preselected as positive with the original version of $\mathrm{AC} 2$, only two CT WT-positive samples were missed with the updated AC2 assay (not sufficient samples remaining for confirmation with ACT). It cannot be excluded that a preselection of samples using the updated AC2 assay would provide similar results when retested in the original $\mathrm{AC} 2$ assay. Both the clinical sensitivity and specificity of the updated AC2 assay for NG detection was $100 \%$.

The recent emergence of $\mathrm{AC} 2$ diagnostic-escape nvCTs [18-25, the present study], spread of the Swedish nvCT escaping detection using the NAATs available from Roche and $\mathrm{Ab}$ bott at that time [4-6, 33], and constantly evolving CT [7, 8], illustrate the necessity of dual target diagnostic CT NAATs and surveillance of the stability of NAAT targets in national and international settings. Furthermore, laboratories should be included in appropriate external quality assessments to detect diagnostic-escape nvCTs and it is imperative to adequately survey and analyse incidence, any unexplained shifts in positivity rates, and/or annual collections of samples diagnosed as negative/equivocal using NAATs with different target(s).

\section{Conclusions}

Widespread implementation of validated, accurate and quality-assured CT dual target NAATs for diagnosis of $\mathrm{CT}$ is crucial internationally. The updated AC2 assay, now including two CT targets and one NG target [27], showed a high clinical sensitivity and specificity, as well as an ideal inclusivity and exclusivity in the detection of WT CT, all described nvCTs [4-6,18-25, the present study], and NG. Nevertheless, international and national surveillance programmes capturing NAAT diagnosticescape variants for $\mathrm{CT}$ as well as other infectious agents are imperative.

\section{Abbreviations}

AC2: Aptima Combo 2; ACT: Aptima Chlamydia trachomatis; CT: C. trachomatis; EU/EEA: European Union/European Economic Area; fg: femtogram; FI-nvCT: Finnish new variant of C. trachomatis; GEQ: Genome equivalent; LGV: Lymphogranuloma venereum; NAAT: Nucleic acid amplification test; NG: Neisseria gonorrhoeae; nvCT: New variant of $C$. trachomatis; PIP: Prolyliminopeptidase; RUO: Research use only; SNP: Single nucleotide polymorphism; STI: Sexually transmitted infection; TC: Target capture; TMA: Transcription-mediated amplification; TV: Trichomonas vaginalis; WHO: World Health Organization; WT: Wild type

\section{Acknowledgements}

We are very grateful to Hologic Inc. (San Diego, USA), particularly Damon Getman, Ashley Nenninger and Philipp Mueller, who provided the updated AC2 kits (as RUO material). Hologic had no role in data collection or interpretation.

\section{Authors' contributions}

$M U, M H, R H, M S$ designed the study. MH, RH, MP, HW, KRJ, CT, MJC, IB, PTL, $\mathrm{SI}$ collected the samples after approving the study design. $\mathrm{MH}, \mathrm{RH}, \mathrm{DG}$ performed the laboratory work. MU, MH, RH analysed the data. MU wrote the first draft of manuscript. All authors were involved in finalising the manuscript and approved the final version of the manuscript.

\section{Funding}

The study was supported by the Örebro County Council Research Committee and the Foundation for Medical Research at Örebro University Hospital, Örebro, Sweden. Open access funding provided by Örebro University.

\section{Availability of data and materials}

The datasets used and/or analysed during the current study are available from the corresponding author on reasonable request.

\section{Ethics approval and consent to participate}

All examined clinical AC2 samples were collected in routine diagnostics (standard care), and no patient identification information was available in the present study. Accordingly, no ethical approval was required.

\section{Consent for publication}

Not applicable.

\section{Competing interests}

The authors declare that they have no competing interests.

\section{Author details}

${ }^{1}$ World Health Organization Collaborating Centre for Gonorrhoea and Other Sexually Transmitted Infections (STIs), National Reference Laboratory for STIS, Department of Laboratory Medicine, Clinical Microbiology, Faculty of Medicine and Health, Örebro University, SE-701 85 Örebro, Sweden. ${ }^{2}$ Department of Virology and Immunology, University of Helsinki and Helsinki University Hospital, HUSLAB, Helsinki, Finland. ${ }^{3}$ Department of Clinical Medicine, Faculty of Medical Sciences, University of Copenhagen, Copenhagen, Denmark. ${ }^{4}$ Department of Clinical Microbiology, Hvidovre University Hospital, Hvidovre, Denmark. ${ }^{5}$ Department of Clinical Microbiology, Turku University Hospital, Turku, Finland. 'Department of Microbiology, Unilabs Laboratory Medicine, Skien, Norway. ${ }^{7}$ National Infection Service, Public Health England, London, UK. ${ }^{8}$ Department of Functional and Laboratory Diagnostics, I. Horbachevsky Ternopil National Medical University, Ternopil, Ukraine. ${ }^{9}$ Hanoi Medical University, National Hospital of

Dermatology and Venereology, Hanoi, Vietnam. ${ }^{10}$ iClinic, Sendai, Japan.

Received: 13 May 2020 Accepted: 11 June 2020

Published online: 16 June 2020

\section{References}

1. Rowley J, Vander Hoorn S, Korenromp E, Low N, Unemo M, Abu-Raddad LJ, et al. Chlamydia, gonorrhoea, trichomoniasis and syphilis: global prevalence and incidence estimates, 2016. Bull World Health Organ. 2019;97:548-62P. 
2. European Centre for Disease Prevention and Control. Surveillance Atlas of Infectious Diseases. https://atlas.ecdc.europa.eu/public/index.aspx (Accessed: May 13, 2020)

3. Lanjouw E, Ouburg S, de Vries HJ, Stary A, Radcliffe K, Unemo M. Background review for the '2015 European guideline on the management of Chlamydia trachomatis infections'. Int J STD AIDS. 2015; 0956462415618838. https://doi.org/10.1177/0956462415618838.

4. Ripa T, Nilsson P. A variant of Chlamydia trachomatis with deletion in cryptic plasmid: implications for use of PCR diagnostic tests. Euro Surveill. 2006; 11(11):E061109.2. https://doi.org/10.2807/esw.11.45.03076-en.

5. Unemo M, Clarke IN. The Swedish new variant of Chlamydia trachomatis Curr Opin Infect Dis. 2011;24:62-9.

6. Herrmann B, Törner A, Low N, Klint M, Nilsson A, Velicko I, et al. Emergence and spread of Chlamydia trachomatis variant, Sweden. Emerg Infect Dis. 2008;14:1462-5.

7. Hadfield J, Harris SR, Seth-Smith HMB, Parmar S, Andersson P, Giffard PM, et al. Comprehensive global genome dynamics of Chlamydia trachomatis show ancient diversification followed by contemporary mixing and recent lineage expansion. Genome Res. 2017;27:1220-9.

8. Harris SR, Clarke IN, Seth-Smith HM, Solomon AW, Cutcliffe LT, Marsh P, et al. Whole-genome analysis of diverse Chlamydia trachomatis strains identifies phylogenetic relationships masked by current clinical typing. Nat Genet. 2012:44:413-9.

9. Silveira MF, Bruni MP, Stauffert D, Golparian D, Unemo M. Prevalence and risk factors associated with Chlamydia trachomatis, Neisseria gonorrhoeae, and Mycoplasma genitalium among women in Pelotas, Southern Brazil. Int J STD AIDS. 2020:31:432-9.

10. Boiko I, Golparian D, Krynytska I, Unemo M. High prevalence of Chlamydia trachomatis, Neisseria gonorrhoeae and particularly Trichomonas vaginalis diagnosed using US FDA-approved Aptima molecular tests and evaluation of conventional routine diagnostic tests in Ternopil, Ukraine. APMIS. 2019; 127:627-34.

11. Cheng A, Kirby JE. Evaluation of the Hologic gen-probe PANTHER, APTIMA combo 2 assay in a tertiary care teaching hospital. Am J Clin Pathol. 2014; 141:397-403.

12. Boyadzhyan B, Yashina T, Yatabe JH, Patnaik M, Hill CS. Comparison of the APTIMA CT and GC assays with the APTIMA combo 2 assay, the Abbott LCX assay, and direct fluorescent-antibody and culture assays for detection of Chlamydia trachomatis and Neisseria gonorrhoeae. J Clin Microbiol. 2004;42: 3089-93.

13. Tabrizi SN, Unemo M, Limnios AE, Hogan TR, Hjelmevoll SO, Garland SM, et al. Evaluation of six commercial nucleic acid amplification tests for detection of Neisseria gonorrhoeae and other Neisseria species. J Clin Microbiol. 2011;49:3610-5.

14. Kowalski RP, Karenchak LM, Raju LV, Ismail N. The verification of nucleic acid amplification testing (gen-probe Aptima assay) for Chlamydia trachomatis from ocular samples. Ophthalmology. 2015;122:244-7.

15. Golparian D, Tabrizi SN, Unemo M. Analytical specificity and sensitivity of the APTIMA Combo 2 and APTIMA GC assays for detection of commensal Neisseria species and Neisseria gonorrhoeae on the Gen-Probe Panther instrument. Sex Transm Dis. 2013;40:175-8.

16. Bristow CC, McGrath MR, Cohen AC, Anderson LA, Gordon KK, Klausner JD. Comparative evaluation of two nucleic acid amplification tests for the detection of Chlamydia trachomatis and Neisseria gonorrhoeae at extragenital sites. Sex Transm Dis. 2017:44:398-400.

17. Rumyantseva T, Golparian D, Nilsson CS, Johansson E, Falk M, Fredlund H, et al. Evaluation of the new AmpliSens multiplex real-time PCR assay for simultaneous detection of Neisseria gonorrhoeae, Chlamydia trachomatis, Mycoplasma genitalium, and Trichomonas vaginalis. APMIS. 2015;123:879-86.

18. Rantakokko-Jalava K, Hokynar K, Hieta N, Keskitalo A, Jokela P, Muotiala A, et al. Chlamydia trachomatis samples testing falsely negative in the Aptima Combo 2 test in Finland, 2019. Euro Surveill. 2019;24(22):1900298 https:/muw.eurosurveillance.org/ content/10.2807/1560-7917.ES.2019.24.22.1900298. PMID: 31164192.

19. Hokynar K, Rantakokko-Jalava K, Hakanen A, Havana M, Mannonen L, Jokela P, et al. The Finnish new variant of Chlamydia trachomatis with a single nucleotide polymorphism in the $23 \mathrm{~S}$ rRNA target escapes detection by the Aptima Combo 2 test. Microorganisms. 2019;7(8):227. https:/doi.org/10.3390/microorganisms7080227.

20. Unemo M, Hansen M, Hadad R, Lindroth $Y$, Fredlund $H$, Puolakkainen $M$, et al. Finnish new variant of Chlamydia trachomatis escaping detection in the Aptima Combo 2 assay also present in Örebro County, Sweden, May
2019. Euro Surveill. 2019;24(26) https://www.eurosurveillance.org/content/1 0.2807/1560-7917.ES.2019.24.26.1900370 PMID: 31266590

21. Johansen TB, Kløvstad H, Rykkvin R, Herrfurth-Erichsen EB, Sorthe J, Njølstad $\mathrm{G}$, et al. The 'Finnish new variant of Chlamydia trachomatis' escaping detection in the Aptima Combo 2 assay is widespread across Norway, June to August 2019. Euro Surveill. 2019;24(42) https://www.eurosurveillance.org/ content/10.2807/1560-7917.ES.2019.24.42.1900592 PMID: 31640843.

22. Unemo M, Getman D, Hadad R, Cole M, Thomson N, Puolakkainen M, et al. Letter to the editor: Chlamydia trachomatis samples testing falsely negative in the Aptima Combo 2 test in Finland, 2019. Euro Surveill. 2019;24(24) https://www.eurosurveillance.org/content/10.2807/1560-7917.ES.2019.24.24.1 900354 PMID: 31213219

23. Weinbaum B, Williams A, Hadad R, Vinluan B, Puolakkainen M, Unemo M, Getman D. Validation of an Aptima-format Finnish new variant of Chlamydia trachomatis (FI-nvCT) surveillance assay, 2019. Euro Surveill. 2020;25(5): 2000038. https://doi.org/10.2807/1560-7917.ES.2020.25.5.2000038.

24. Hadad R, Jensen JS, Westh H, Grønbaek I, Schwartz LJ, Nielsen L, et al. A Chlamydia trachomatis 23 S rRNA G1523A variant escaping detection in the Aptima Combo 2 assay (Hologic) was widespread across Denmark in JulySeptember 2019 [published online ahead of print, 2020 Mar 23]. APMIS. 2020. https://doi.org/10.1111/apm.13043.

25. Roberts DJ, Davis GS, Cole MJ, Naik D, Maru H, Woodford N, et al. Prevalence of new variants of Chlamydia trachomatis escaping detection by the Aptima Combo 2 assay, England, June to August 2019. Euro Surveill. 2019;24(38) https://www.eurosurveillance.org/content/10.2807/1560-7917. ES.2019.24.38.1900557 PMID: 31552817..

26. ECDC. Rapid risk assessment: Chlamydia trachomatis false-negative test results by Aptima Combo 2 CT/NG assay (Hologic) in the EU/EEA. Stockholm: European Centre for Disease Prevention and Control; 2019. 17. 06.

27. Getman D. Mechanism of F-nvCT effect on AC2 performance, design and validation of F-nvCT surveillance IUO assay. IUSTI 2019 European congress, 5-7 September 2019, Tallinn, Estonia.

28. Unemo M, Golparian D, Sánchez-Busó L, Grad Y, Jacobsson S, Ohnishi M, et al. The novel 2016 WHO Neisseria gonorrhoeae reference strains for global quality assurance of laboratory investigations: phenotypic, genetic and reference genome characterization. J Antimicrob Chemother. 2016;71:3096108.

29. Jacobsson S, Boiko I, Golparian D, Blondeel K, Kiarie J, Toskin I, et al. WHO laboratory validation of Xpert CT/NG and Xpert TV on the GeneXpert system verifies high performances. APMIS. 2018;126:907-12.

30. Frølund $M$, Lidbrink $P$, Wikström $A$, Cowan $S$, Ahrens $P$, Jensen JS. Urethritisassociated pathogens in urine from men with non-gonococcal urethritis: a case-control study. Acta Derm Venereol. 2016;96:689-94.

31. Priest D, Ong JJ, Chow EPF, Tabrizi S, Phillips S, Bissessor M. Neisseria gonorrhoeae DNA bacterial load in men with symptomatic and asymptomatic gonococcal urethritis. Sex Transm Infect. 2017;93:478-81.

32. Chow EP, Tabrizi SN, Phillips S, Lee D, Bradshaw CS, Chen MY, et al. Neisseria gonorrhoeae bacterial DNA load in the pharynges and saliva of men who have sex with men. J Clin Microbiol. 2016;54:2485-90.

33. Unemo M, Seth-Smith HMB, Cutcliffe LT, Skilton RJ, Barlow D, Goulding D, et al. The Swedish new variant of Chlamydia trachomatis: genome sequence, morphology, cell tropism and phenotypic characterization. Microbiology. 2010;156(Pt 5):1394-404.

\section{Publisher's Note}

Springer Nature remains neutral with regard to jurisdictional claims in published maps and institutional affiliations. 